\title{
Investigation of Potential Drug-Drug Interactions between Peficitinib (ASP015K) and Methotrexate in Patients with Rheumatoid Arthritis
}

\author{
Tong Zhu ${ }^{1}$. Selina Moy $^{2} \cdot$ Udaya Valluri $^{1} \cdot$ Ying Cao $^{1} \cdot$ Wenhui Zhang $^{1} \cdot$ Taiji Sawamoto $^{3} \cdot$ Vishala Chindalore $^{4}$. \\ Bola Akinlade ${ }^{1,5}$
}

Published online: 26 June 2020

(c) The Author(s) 2020

\begin{abstract}
Background Methotrexate is frequently used to treat rheumatoid arthritis. Peficitinib (ASP015K; Smyraf ${ }^{\circledR}$ ), an oral Janus kinase inhibitor indicated for the treatment of rheumatoid arthritis, may be coadministered with methotrexate.

Objective The objective of this study was to investigate potential drug-drug interactions of peficitinib with methotrexate and the short-term safety of coadministration.

Patients and Methods This phase I, open-label, single-sequence study included patients with rheumatoid arthritis taking a stable dose of methotrexate. Patients received their prescribed methotrexate dose (Day 1) and then peficitinib (100 mg) twice daily from Day 3 until the morning of Day 9; a second methotrexate dose was coadministered with peficitinib on Day 8. Serial blood samples were collected for methotrexate concentration after dosing on Days 1 (methotrexate alone) and 8 (methotrexate plus peficitinib) and for peficitinib concentration after dosing on Days 7 (peficitinib alone) and 8 (methotrexate plus peficitinib). Pre-dose concentrations of peficitinib were measured (Days 3-8).

Results Peficitinib concentrations reached steady state on Day 5. Administration of peficitinib did not result in changes to methotrexate area under the concentration-time curve from time zero to infinity or maximum observed concentration following a methotrexate dose (15-25 mg), and there was no significant effect of methotrexate (15-25 mg) on peficitinib area under the concentration-time curve within a 12-hour dosing interval. There were no new tolerability or safety signals after coadministration of peficitinib and methotrexate. One patient experienced two serious adverse events and withdrew from the study without receiving peficitinib.
\end{abstract}

Conclusions Pharmacokinetic results showed no significant interactions between peficitinib and methotrexate.

ClinicalTrials.gov Identifier NCT01754805.

Electronic supplementary material The online version of this article (https://doi.org/10.1007/s40261-020-00937-z) contains supplementary material, which is available to authorized users.

Tong Zhu

tong.zhu@astellas.com

1 Astellas Pharma Global Development, Northbrook, IL, USA

2 Astellas Research Institute of America, LLC, Northbrook, IL, USA

3 Astellas Pharma Inc., Tsukuba, Ibaraki, Japan

4 Pinnacle Research Group/Anniston Medical Clinic, Anniston, AL, USA

5 Present Address: Regeneron Pharmaceuticals, Inc., Tarrytown, NY, USA

\section{Key Points}

As methotrexate and the Janus kinase inhibitor peficitinib may be coadministered in patients with rheumatoid arthritis, it is important to examine any potential drug-drug interactions.

This phase I, open-label, short-term study demonstrated that the two drugs do not affect the pharmacokinetic profile of each other in patients with rheumatoid arthritis.

The coadministration of methotrexate and peficitinib appeared well tolerated, and no abnormalities in laboratory findings, vital signs, or electrocardiogram results were observed. 


\section{Introduction}

Rheumatoid arthritis (RA) is an inflammatory autoimmune disease, with a prevalence of $0.5-1 \%$ worldwide [1]. Guidelines recommend conventional synthetic disease-modifying antirheumatic drugs (DMARDs), such as methotrexate, as first-line treatment in patients with established RA [2, 3]. Methotrexate, in particular, is often considered an anchor drug for treating patients with RA, both alone and in combination with other medications [2, 3]. Despite advances over the past decade, currently available therapies for RA may not be efficacious or tolerable for some patients $[1,4]$, and there is a need for new targeted therapies to treat this disease.

The Janus kinase (JAK) family of enzymes (JAK1, JAK2, JAK3, and tyrosine kinase 2) plays a key role in cytokine signaling and has been identified as a target for new RA therapies [5]. JAK1, JAK2, and tyrosine kinase 2 are expressed ubiquitously; however, JAK3 is mainly expressed in lymphocytes (e.g., natural killer cells, T cells) $[6,7]$. Following interleukin-2 stimulation, JAK3 phosphorylates and activates the signal transducer and activator of transcription-5, which then plays a role in T-cell activation $[8,9]$. Because of its role in erythropoiesis, JAK2 inhibition is associated with several hematologic adverse events (AEs) [10]. Agents that selectively inhibit JAK1 and/or 3 could thus minimize JAK2-related hematopoietic AEs, such as anemia $[11,12]$.

Several JAK inhibitors, including tofacitinib, baricitinib, and peficitinib, have now been licensed for the treatment of patients with RA [13, 14]. Peficitinib (ASP015K; Smyraf $\left.{ }^{\circledR}\right)$ is a pan-JAK inhibitor with half-maximal inhibitory concentration $\left(\mathrm{IC}_{50}\right)$ values of $3.9,5.0,0.7$, and $4.8 \mathrm{nM}$ for JAK1, JAK2, JAK3, and tyrosine kinase 2, respectively [15]. The 2017 European League Against Rheumatism recommendations include JAK inhibitors or biological DMARDs for use in patients who do not respond to first-line therapy with conventional synthetic DMARDs [3], and coadministration of peficitinib with methotrexate is likely to be common in clinical practice. A drug-drug interaction study is therefore important to elucidate the potential for interactions between peficitinib and methotrexate when these drugs are coadministered.

In humans, over $80 \%$ of a methotrexate dose is excreted unchanged in urine over $24 \mathrm{~h}$; within the kidney, the drug is filtered by the glomeruli and then undergoes tubular secretion and reabsorption [16]. Methotrexate is partly oxidized by hepatic aldehyde oxidase to form 7-hydroxymethotrexate [17]. Methotrexate is a substrate of several membrane transporters located in the renal tubule, including multidrug resistance-associated proteins 2 and 4 (MRP2/4), breast cancer resistance protein (BCRP, also known as $\mathrm{ABCG} 2$ ), and organic anion transporters 1 and $3(\mathrm{OAT} 1 / 3)[16,18-20]$. Breast cancer resistance protein is also expressed in the intestinal epithelium and has a role in methotrexate absorption [18, 21]. The main clearance mechanism for peficitinib is hepatic metabolism, predominantly via sulfate and methyl conjugation [14]. In healthy male subjects, over $65 \%$ of an oral dose has been reported to undergo metabolism [22]. Approximately 37\% of the oral dose was excreted in urine (14\% of the dose was excreted unchanged), and approximately $57 \%$ was excreted in feces suggesting possible biliary excretion [22].

Here, we report findings from a clinical study, for which the primary objective was to evaluate the effect of peficitinib $100 \mathrm{mg}$ twice daily (BID) on the pharmacokinetics of once-weekly oral methotrexate $15-25 \mathrm{mg}$. Secondary objectives were to evaluate the effect of methotrexate on steady-state pharmacokinetics of peficitinib, and to evaluate the safety and tolerability of coadministration of the two drugs in patients with RA. The clinical study was supported by in vitro studies that investigated the potential inhibitory effects of peficitinib on MRP2/4-, BCRP-, and OAT1/3mediated transport; these studies identified an inhibitory effect of peficitinib on BCRP and OAT3 (Electronic Supplementary Material).

\section{Patients and Methods}

\subsection{Patients}

Sixteen male or female patients with RA and currently receiving methotrexate were to be enrolled in the study. Female patients of child-bearing potential were not allowed to participate. Patients were eligible for the study if they were aged 18-65 years, had a body mass index $\leq 35 \mathrm{~kg} / \mathrm{m}^{2}$, and had a clinical diagnosis of RA for $\geq 6$ months before screening. Patients had to have been treated with methotrexate at a stable dose of $15-25 \mathrm{mg}$ once weekly for $\geq 28$ days before study entry. Women who were enrolled had to be $\geq 2$ years post-menopausal or surgically sterile. Men had to agree to sexual abstinence and/or use a highly effective method of birth control during the study period and for 60 days after the last dose of the study drug. Patients were excluded if they were currently receiving a biologic DMARD; had received a live virus vaccination $\leq 30$ days prior to study drug administration; or had any clinically significant illness, medical condition, or laboratory abnormality that the investigator deemed significant enough to prevent the patient from participating. Patients were also excluded if they had any of the following: a history of anemia (as defined by hemoglobin $<12 \mathrm{~g} / \mathrm{dL}$ ); absolute neutrophil count $<2500$ cells $/ \mathrm{mm}^{3}$; liver enzyme test abnormalities; 
or alanine aminotransferase, aspartate aminotransferase, or total bilirubin two or more times the upper limit of normal.

\subsection{Study Design}

This was an open-label, single-sequence, drug interaction study with oral peficitinib (100 mg) BID and once-weekly oral methotrexate (15-25 mg) [ClinicalTrials.gov Identifier: NCT01754805]. The study was conducted at a single site in the USA (Pinnacle Research Group/Anniston Medical Clinic, Anniston, AL, USA) from January 2010 to March 2010. The study protocol and the consent form were approved by an independent institutional review board (Quorum Review Institutional Review Board, Seattle, WA, USA) and conducted in accordance with the Declaration of Helsinki and applicable laws and regulations. All patients provided written informed consent.

Three treatment periods were defined in this drug-drug interaction study (Fig. 1). Patients received all treatments in the fed state, and study drugs were swallowed whole with $240 \mathrm{~mL}$ of water. On Day 1, patients received their usual prescribed morning methotrexate dose. Starting on Day 3 through to the morning of Day 9, patients received peficitinib $100 \mathrm{mg}$ BID with doses approximately $12 \mathrm{~h}$ apart; a second prescribed dose of methotrexate in combination with peficitinib was given on the morning of Day 8. Timing of administration of doses was consistent for the duration of the study [23, 24]. The 100-mg BID dose of peficitinib selected for this study was expected to provide $33 \%$ higher exposure [area under the concentration-time curve (AUC)] than $150 \mathrm{mg}$ once daily, and $100 \%$ higher exposure than $100 \mathrm{mg}$ once daily, thereby maximizing the potential to detect drug-drug interactions and assess tolerability.

\subsection{Assessments}

Blood samples (4 mL) for determining concentrations of methotrexate and its metabolite, 7-hydroxymethotrexate, were collected in 4-mL lithium heparin vacutainer tubes pre-dose on Day 1 (methotrexate alone) and Day 8 (methotrexate plus peficitinib) and at $0.25,0.5,1,1.5,2,3,4,6$, $8,12,24,36$, and $48 \mathrm{~h}$ post-dose on each day. Blood samples $(2 \mathrm{~mL})$ for determining peficitinib concentrations were collected in 2-mL K3 EDTA vacutainer tubes prior to the morning dose $\left(C_{\text {trough }}\right)$ on Days $3,4,5,6,7,8$, and 9. Blood samples $(2 \mathrm{~mL})$ were also collected at $0.5,1,1.5,2,3,4,6$, 8 , and 12 h post-dose on Day 7 (peficitinib alone) and Day 8 (methotrexate plus peficitinib). Plasma was harvested within $60 \mathrm{~min}$ of blood sampling and stored at $-80^{\circ} \mathrm{C}$ prior to analysis with liquid chromatography-tandem mass spectrometry.

\subsection{Bioanalytical Methods}

Peficitinib was extracted by supported liquid extraction. Plasma samples $(50 \mu \mathrm{L})$ were combined with an internal standard, d3-ASP015K, and extracted using methyl-tertbutyl ether under basic conditions. The extraction was performed in a 96-well format using supported liquid extraction plates. The elution solvent (methyl-tert-butyl ether) was evaporated. The residue was reconstituted and submitted to analysis by liquid chromatography-tandem mass spectrometry. Separation was achieved on a Betasil Silica-100 column ( $5 \mu \mathrm{m}, 50 \times 3 \mathrm{~mm}$; Thermo Scientific, Waltham, MA, USA) with a mobile phase composed of water:acetonitrile:acetic acid:trifluoroacetic acid. The analytes were monitored on a Sciex API4000 (Applied Biosystems, Redwood City, CA, USA) using a positive ion spray. The transitions monitored were $\mathrm{m} / \mathrm{z} 327.14 \rightarrow 160.20$ for peficitinib and $\mathrm{m} / \mathrm{z}$ $329.99 \rightarrow 163.20$ for the internal standard. The concentrations of peficitinib were calculated using peak area ratios (analyte/internal standard). The calibration curves were generated using a linear regression with a weighting of $1 / x^{2}$ and the range was 0.250 (lower limit of quantification) to $250 \mathrm{ng} / \mathrm{mL}$ (upper limit of quantification). The precision (\% coefficient of variation) was $<8.6 \%$ and the accuracy ranged from -4.0 to $8.2 \%$ relative error.

Analysis of plasma concentrations of methotrexate and 7-hydoxymethotrexate was performed using a validated
Fig. 1 Treatment periods used for drug-drug interaction study and analyses. BID twice daily. Patients received their usual prescribed methotrexate dose (15-25 mg) on Day 1 and a second prescribed dose on Day 8

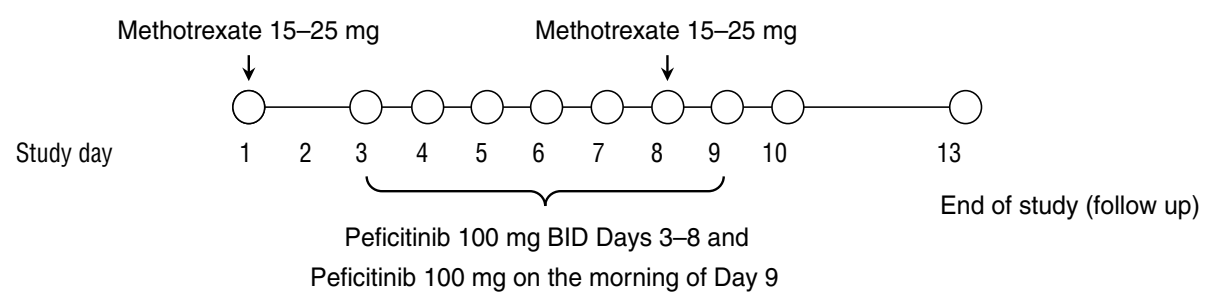

\begin{tabular}{lcc}
\hline & \multicolumn{2}{c}{ Study day } \\
\cline { 2 - 3 } Treatment period & Start & Stop \\
\hline $\begin{array}{l}\text { Methotrexate alone }(\mathrm{n}=15) \\
\text { Peficitinib alone }(\mathrm{n}=14)\end{array}$ & Day 1 (after methotrexate dose) & Day 3 (before peficitinib dose) \\
Methotrexate + peficitinib $(\mathrm{n}=14)$ & Day 8 (after methotrexate dose) & Day 8 (before methotrexate dose) \\
\end{tabular}


high-performance liquid chromatography coupled with tandem mass spectrometric detection method by $Q^{2}$ Solutions (Bioanalytical Laboratories, Ithaca, New York, NY, USA [formerly Advion Bioservices]). The lower limit of quantification for methotrexate and 7-hydroxymethotrexate was $1.0 \mathrm{ng} / \mathrm{mL}$ with $200 \mu \mathrm{L}$ of plasma via a solid phase extraction (method on file). Concentrations were calculated using peak area ratios (analyte/internal standard). The calibration curves were generated using a linear regression line with a weighting of $1 / x^{2}$ and the ranges for methotrexate and 7-hydroxymethotrexate were $1-1000 \mathrm{ng} / \mathrm{mL}$ and $1-150 \mathrm{ng} / \mathrm{mL}$, respectively. The precision (\% coefficient of variation) was $<5.2 \%$ and the accuracy ranged from -7.0 to $0.7 \%$ relative error.

No significant interference was observed in the chromatographic region of interest for the analytes in their respective methods when their respective quality control samples were fortified with the co-administered analyte(s). Study samples measuring over the upper limit of quantification were repeated using up to 20 -fold dilution for peficitinib and up to 10 -fold dilution for both methotrexate and 7-hydroxymethotrexate.

\subsection{Pharmacokinetic Analysis}

Pharmacokinetic parameters were calculated using noncompartmental methods (WinNonlin ${ }^{\circledR}$ Professional, version 5.3; Pharsight Corporation, Mountain View, CA, USA) from individual patient plasma concentration-time profiles. For methotrexate, the maximum observed concentration $\left(C_{\max }\right)$, time to reach $C_{\max }\left(t_{\max }\right)$, and AUC from time zero to last measurable concentration $\left(\mathrm{AUC}_{\text {last }}\right)$ and from time zero to infinity $\left(\mathrm{AUC}_{\mathrm{inf}}\right)$ were calculated after each dose of methotrexate on Days 1 and 8, and the half-life calculated. Plasma concentrations of its active metabolite 7-hydroxymethotrexate were also measured. As patients received individualized doses of methotrexate, pharmacokinetic parameters were normalized by dose. For peficitinib, $C_{\max }, t_{\max }$, and AUC within a 12-h dosing interval $\left(\mathrm{AUC}_{12}\right)$ on Days 7 and 8 were assessed. The primary study variables were $\mathrm{AUC}_{\mathrm{inf}}$ and $C_{\max }$ of methotrexate. Area under the concentration-time curves were calculated using the linear-log trapezoidal method. The linear trapezoidal method was used when concentrations were increasing and the logarithmic trapezoidal method was used when concentrations were decreasing.

\subsection{Safety Assessments}

Safety was assessed by monitoring of reported AEs, 12-lead electrocardiogram measurements, vital signs, clinical laboratory evaluations, and physical examinations. A serious $\mathrm{AE}$ (SAE) was defined as one that was considered medically important, was life threatening, or resulted in any of the following: death, persistent or significant disability/incapacity, a congenital anomaly or birth defect, hospitalization of the patient, or prolongation of hospitalization.

\subsection{Statistical Analyses}

No formal power analysis was performed to determine the sample size for the study. Based on clinical judgment, a sample size of 16 patients with RA was thought to be reasonable to assess the effect of peficitinib on the pharmacokinetics of methotrexate and to evaluate the safety and tolerability of coadministration of peficitinib and methotrexate. All data analyses were performed using $\mathrm{SAS}^{\circledR}$ version 9.1.

To assess the effect of multiple doses of peficitinib on the pharmacokinetics of methotrexate, a $90 \%$ confidence interval (CI) was constructed for the ratio of geometric means of plasma $\mathrm{AUC}_{\text {inf }}$ and $C_{\max }$ of methotrexate with peficitinib (Day 8 ) vs methotrexate alone (Day 1), based on a mixedeffects model of natural logarithm-transformed $\mathrm{AUC}_{\mathrm{inf}}$ and $C_{\max }$, with treatment as a fixed effect and patient as a random effect. The least-square mean difference of Day 8 vs Day 1 (and associated 90\% CI) were back-transformed to the original scale to obtain the geometric mean and $90 \% \mathrm{CI}$ for these ratios. Peficitinib was deemed to have no effect on the pharmacokinetics of methotrexate if the $90 \%$ CI for both $\mathrm{AUC}_{\text {inf }}$ and $C_{\max }$ ratios fell between 0.80 and 1.25.

To assess the effect of methotrexate on the steady-state pharmacokinetics of peficitinib, a similar model was used, and the $90 \%$ CI was constructed for the geometric mean ratio of plasma $\mathrm{AUC}_{12}$ and $C_{\max }$ of peficitinib with methotrexate (Day 8) vs peficitinib alone (Day 7). The attainment of steady state for peficitinib was assessed using $C_{\text {trough }}$ on Days 4-8. Using an analysis of variance model of natural logarithm-transformed $C_{\text {trough }}$, with day as a fixed effect and patient as a random effect, a contrast test was performed for Day 4 vs the average of trough values on Days 5-8. Steady state was concluded if the $p$ value for the contrast was $>0.05$. If steady state was not established, the contrast was repeated with the next day vs the average of the trough concentrations beyond the next day.

Safety variables were summarized using frequency and descriptive statistics. Adverse events were coded using the Medical Dictionary for Regulatory Activities, version 11.1.

\section{Results}

\subsection{Patient Disposition and Demographics}

A total of 15 patients enrolled in the study and were included in the safety analysis. Of the 15 patients, ten received two 15-mg once-weekly doses of methotrexate during the study and the remainder received higher doses (i.e., $17.5 \mathrm{mg}$ 


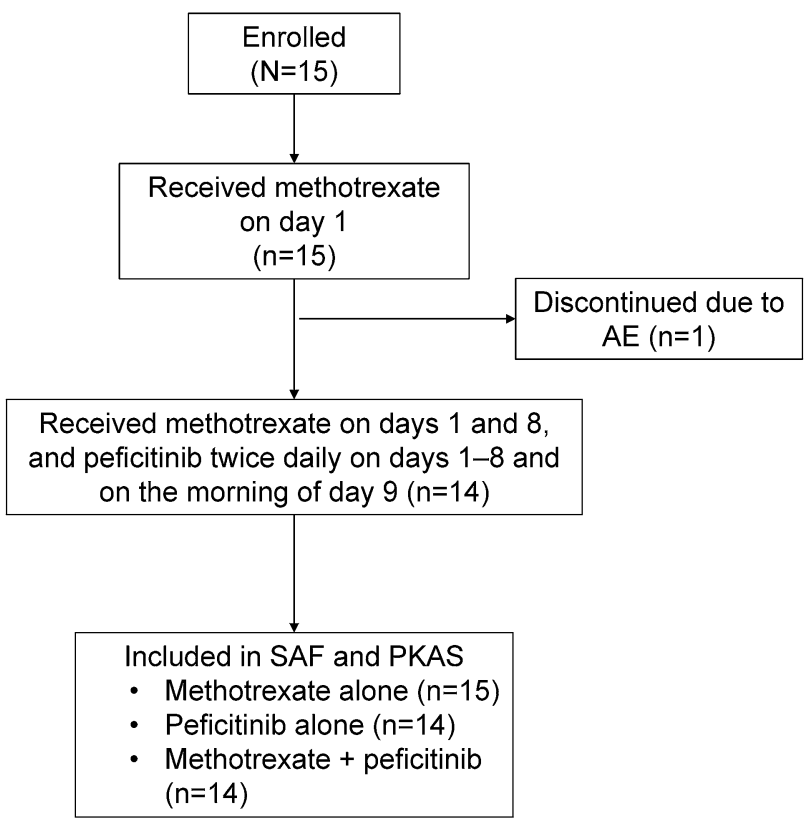

Fig. 2 Patient flow through the study. $A E$ adverse event, $P K A S$ pharmacokinetic analysis set, $S A F$ safety analysis set

[ $n=2], 20 \mathrm{mg}[n=2], 25 \mathrm{mg}[n=1]$ once weekly). One female patient withdrew from the study because of an SAE on Day 1 (see Sect. 3.3), leaving a total of 14 patients who completed the study (Fig. 2). Baseline demographics are shown in Table 1. In addition to drugs used by most patients (such as detoxifying agents, folic acid, systemic anti-psoriatic drugs, other immunosuppressants, and gynecological agents), the most frequent concomitant medications were anilides (e.g., acetanilides, benzanilides, salicylanilides; $60 \%$ of patients) followed by calcium in combination with other drugs, locally acting corticosteroids, glucocorticoids, and proton pump inhibitors ( $40 \%$ of patients for each).

\subsection{Pharmacokinetic Measurements}

\subsubsection{Peficitinib as a Potential Perpetrator of Pharmacokinetic Drug-Drug Interactions with Methotrexate}

The mean ( \pm standard error) dose-normalized plasma concentration-time profiles of methotrexate (potential victim drug) were similar with (Day 8) and without (Day 1) coadministration of peficitinib (potential perpetrator drug) (Fig. 3a). Pharmacokinetic results for methotrexate on Days 1 and 8 are shown in Tables 2 and 3. The $t_{\max }$ and dose-normalized $C_{\max }, \mathrm{AUC}_{\text {inf }}$, and AUC from time zero to last measurable concentration for methotrexate were comparable when administered with or without peficitinib (Tables 2, 3). Statistical analyses confirmed that there was
Table 1 Summary of demographics and baseline characteristics of all patients enrolled $^{\mathrm{a}}$

\begin{tabular}{ll}
\hline Characteristics & Value \\
\hline Number of patients & 15 \\
Sex, $n(\%)$ & \\
Men & $4(27)$ \\
Women & $11(73)$ \\
Race, $n(\%)$ & $14(93)$ \\
White & $1(7)$ \\
Black or African American & \\
Ethnicity, $n(\%)$ & $13(87)$ \\
Not Hispanic or Latino & $2(13)$ \\
Hispanic or Latino & \\
Age (years) & $56.1(8.0)$ \\
Mean (SD) & $58.0(35.0-64.0)$ \\
Median (range) & \\
Weight ${ }^{\mathrm{b}}(\mathrm{kg})$ & $69.7(12.4)$ \\
Mean (SD) & $70.5(44.5-86.8)$ \\
Median (range) & \\
Body mass index ${ }^{\mathrm{b}}\left(\mathrm{kg} / \mathrm{m}^{2}\right)$ & $25.0(3.1)$ \\
Mean (SD) & $25.9(18.0-28.7)$ \\
Median (range) &
\end{tabular}

Patients must have been receiving a stable dose of methotrexate for $\geq 28$ days before screening; during the study, they received their stable dose of methotrexate (15-25 mg once weekly; $15 \mathrm{mg}[n=10]$, $17.5 \mathrm{mg}[n=2], 20 \mathrm{mg}[n=2], 25 \mathrm{mg}[n=1])$ on Day 1 (single dose) and Day 8 (single dose coadministered with peficitinib). Patients received peficitinib $100 \mathrm{mg}$ BID on Day 3 through to the morning of Day 9

$B I D$ twice daily, $S D$ standard deviation

${ }^{a}$ Safety analysis set, which included all patients who received at least one dose of the study drug. This included the one patient who discontinued before receiving any peficitinib

${ }^{\mathrm{b}}$ Weight at screening visit (used for the body mass index)

no significant effect of multiple doses of peficitinib on the pharmacokinetics of methotrexate (Table 4). The 90\% CI for the geometric mean ratio of methotrexate $C_{\max }$ and $\mathrm{AUC}_{\text {inf }}$ on Day 8 (methotrexate plus peficitinib) vs Day 1 (methotrexate alone) fell within the predefined no-effect range of $80-125 \%$ (Table 4 ).

Changes in methotrexate pharmacokinetic parameters (dose-normalized $C_{\max }$ and $\mathrm{AUC}_{\text {inf }}$ ) by peficitinib coadministration varied fourfold between individual patients. However, there was no trend toward a higher peficitinib exposure being associated with greater changes in methotrexate pharmacokinetic parameters (data not shown). Coadministration of methotrexate and peficitinib decreased the metabolite ratio, defined as $\mathrm{AUC}_{\text {inf }}$

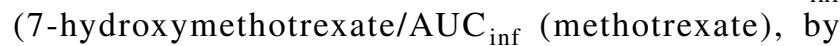
approximately $27 \%$ (Table 4 ). In addition, half-life values 

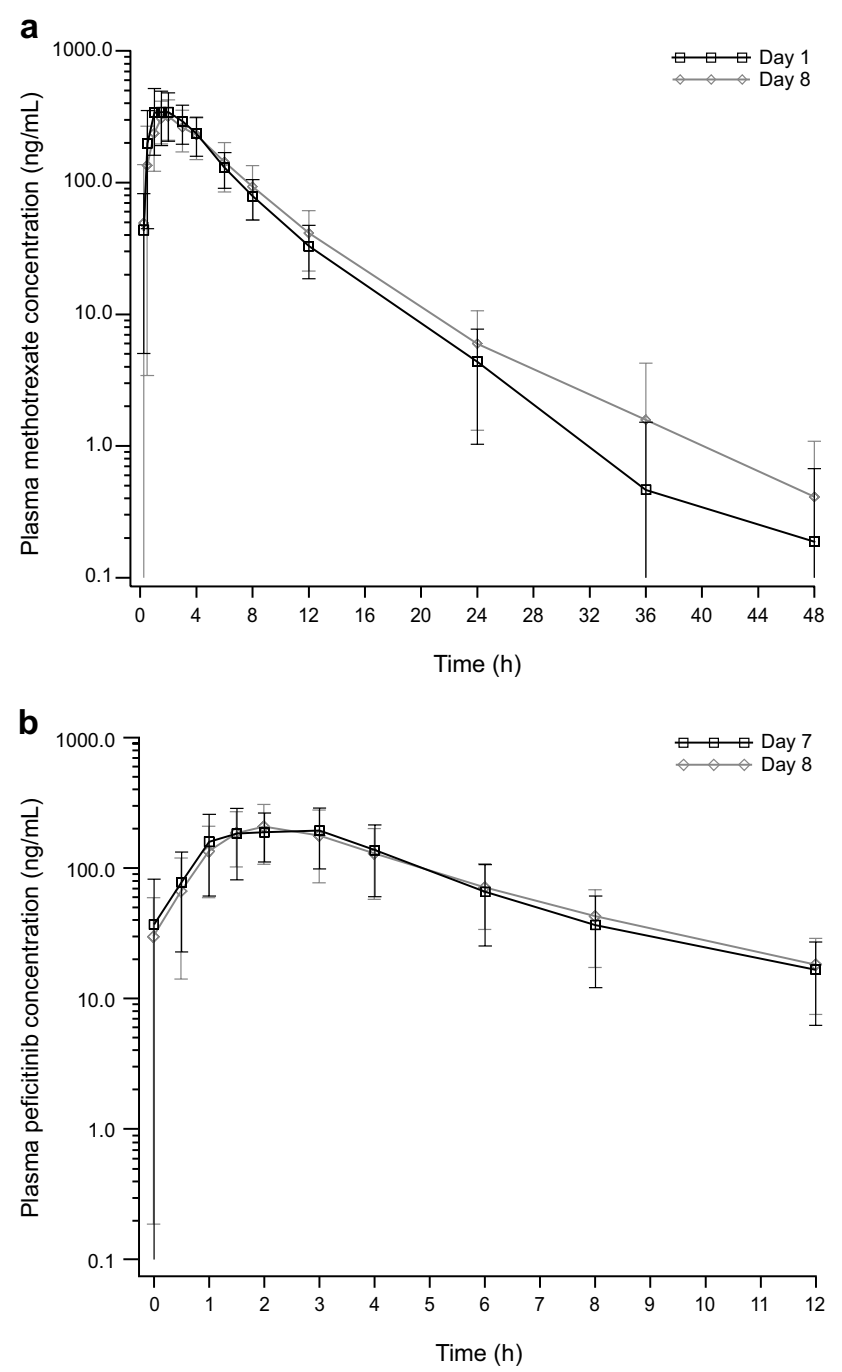

Fig. 3 a Mean ( \pm standard deviation) dose-normalized plasma methotrexate concentration-time profile on Days 1 (alone) and 8 (in combination with peficitinib). b Mean ( \pm standard deviation) plasma peficitinib concentration vs time profile on Days 7 (peficitinib alone) and 8 (in combination with methotrexate). Concentrations below the lower limit of quantification (methotrexate $=1 \mathrm{ng} / \mathrm{mL}$; peficitinib $=0.25 \mathrm{ng}$ / $\mathrm{mL}$ ) were set to 0

were larger for 7-hydroxymethotrexate than methotrexate, and were larger for both with coadministration of peficitinib (Table 3).

\subsubsection{Methotrexate as a Potential Perpetrator of Pharmacokinetic Drug-Drug Interactions with Peficitinib}

Peficitinib plasma concentrations reached steady state by Day 5 as determined by the contrast test of peficitinib $C_{\text {trough }}$ values, which showed no statistically significant difference on Day 5 vs the average $C_{\text {trough }}$ on Days 6-8 $(p=0.8309)$. This result was as expected owing to the relatively short half-life of peficitinib. Mean ( \pm standard error) plasma concentrations-time profiles of peficitinib (potential victim drug) with (Day 8) and without (Day 7) coadministration of methotrexate (potential perpetrator drug) are presented in Fig. 3b. Summaries of peficitinib plasma pharmacokinetic parameters $\left(\mathrm{AUC}_{12}, C_{\max }, t_{\max }, C_{\text {trough }}\right)$ with and without methotrexate are shown in Table 5. Statistical analyses showed that there was no significant effect of methotrexate on peficitinib $\mathrm{AUC}_{12}$. The $90 \% \mathrm{CI}$ for the geometric mean ratio of peficitinib $\mathrm{AUC}_{12}$ on Day 8 (methotrexate plus peficitinib) vs Day 7 (peficitinib alone) fell within the noeffect reference range of $80-125 \%$ (Table 4). The geometric mean ratio of $C_{\max }$ showed that there was an $8 \%$ decrease of peficitinib $C_{\max }$ with methotrexate coadministration (Day 8) compared with peficitinib administered alone (Day 7), and the lower bound of the $90 \%$ CI (78.2\%) was slightly outside the reference range (80-125\%) (Table 4).

\subsection{Safety}

Of 15 patients, five (33.3\%) experienced one or more AEs when administered methotrexate 15-25 mg once weekly alone. Seven of $14(50.0 \%)$ patients experienced one or more AEs when administered methotrexate $15-25 \mathrm{mg}$ once weekly plus peficitinib $100 \mathrm{mg}$ BID, and 7 of 14 (50.0\%) patients experienced one or more AEs when administered peficitinib $100 \mathrm{mg}$ BID alone. One patient experienced two SAEs before receiving peficitinib; one event (urinary tract infection) occurred before any dosing (methotrexate or peficitinib), and one event (gastroenteritis) occurred after the patient received methotrexate on Day 1 . This patient was discontinued from the study. The most frequently reported AEs in this study were gastrointestinal disorders (Table 6), with flatulence being reported solely with peficitinib use and nausea solely with methotrexate alone. Overall, 6 of 15 (40.0\%) patients experienced AEs that were considered related to the study drug: 2 of $15(13.3 \%)$ patients during the methotrexate-alone period, 4 of 14 (28.6\%) patients in the peficitinib-alone period, and 2 of 14 (14.3\%) patients during the methotrexate plus peficitinib period.

There were no clinically meaningful changes from baseline for any of the clinical laboratory parameters (hematology, biochemistry, or urinalysis). No abnormal clinical laboratory values were reported as AEs and no patient discontinued from the study because of an abnormal laboratory value. Few potentially clinically relevant changes from baseline were observed in vital signs. Overall, there were 13 incidences of changes in systolic blood pressure (methotrexate alone, 4; peficitinib alone, 3; methotrexate plus peficitinib, 6), 24 incidences of changes in diastolic blood pressure (methotrexate alone, 7; peficitinib alone, 9; methotrexate plus peficitinib, 8), and 12 incidences of either high or low pulse rates (methotrexate alone, 2; peficitinib alone, 
Table 2 Summary of dose-normalized pharmacokinetic parameters for methotrexate

\begin{tabular}{|c|c|c|}
\hline Parameter & $\begin{array}{l}\text { Day } 1 \\
\text { Methotrexate }{ }^{\mathrm{a}} \text { alone } \\
(n=15)\end{array}$ & $\begin{array}{l}\text { Day } 8 \\
\text { Methotrexate + peficitinib } \\
(n=14)^{\mathrm{b}}\end{array}$ \\
\hline \multicolumn{3}{|c|}{$\mathrm{AUC}_{\text {inf }} /$ dose $(\mathrm{h} \bullet \mathrm{ng} / \mathrm{mL}) / \mathrm{mg}$} \\
\hline Mean (SD) & $122.5(31.5)$ & $127.9(40.9)$ \\
\hline Median (range) & $130.1(57.7-163.6)$ & $137.4(53.4-175.9)$ \\
\hline \multicolumn{3}{|c|}{$C_{\max } / \mathrm{dose}(\mathrm{ng} / \mathrm{mL}) / \mathrm{mg}$} \\
\hline Mean (SD) & $24.0(7.2)$ & $21.8(6.0)$ \\
\hline Median (range) & $25.0(7.7-38.8)$ & $23.0(8.1-31.7)$ \\
\hline \multicolumn{3}{|c|}{$\mathrm{AUC}_{\text {last }} / \mathrm{dose}(\mathrm{h} \bullet \mathrm{ng} / \mathrm{mL}) / \mathrm{mg}$} \\
\hline Mean (SD) & $121.6(31.5)$ & $126.7(40.9)$ \\
\hline Range & $129.2(56.2-163.0)$ & $136.2(52.0-175.1)$ \\
\hline
\end{tabular}

$A U C$ area under the concentration-time curve, $A U C_{i n f}$ area under the concentration-time curve from time zero to infinity, $A U C_{\text {last }}$ area under the concentration-time curve from time zero to last measurable concentration, $B I D$ twice daily, $C_{\text {max }}$ maximum observed concentration, $h$ hours, $S D$ standard deviation

${ }^{a}$ Methotrexate (15-25 mg once weekly; $\left.15 \mathrm{mg}[n=10], 17.5 \mathrm{mg}[n=2], 20 \mathrm{mg}[n=2], 25 \mathrm{mg}[n=1]\right)$ was administered on Days 1 and 8. Peficitinib (100 mg) was administered BID on Days 3-8, inclusive, and on the morning of Day 9

${ }^{\mathrm{b}}$ One patient experienced two serious adverse events and withdrew from the study; this patient did not receive peficitinib

Table 3 Summary of pharmacokinetic parameters for methotrexate and the metabolite 7-hydroxymethotrexate on Day 1 (alone) and Day 8 (in combination with peficitinib)

\begin{tabular}{|c|c|c|c|c|}
\hline \multirow[t]{2}{*}{ Parameter } & \multicolumn{2}{|l|}{ Methotrexate } & \multicolumn{2}{|l|}{ 7-Hydroxymethotrexate } \\
\hline & $\begin{array}{l}\text { Day } 1 \\
\text { Methotrexate alone } \\
(n=15)\end{array}$ & $\begin{array}{l}\text { Day } 8 \\
\text { Methotrexate + peficitinib } \\
(n=14)^{\mathrm{b}}\end{array}$ & $\begin{array}{l}\text { Day } 1 \\
\text { Methotrexate alone }{ }^{\mathrm{a}} \\
(n=15)\end{array}$ & $\begin{array}{l}\text { Day } 8 \\
\text { Methotrexate }+ \text { peficitinib }^{\mathrm{a}} \\
(n=14)^{\mathrm{b}}\end{array}$ \\
\hline \multicolumn{5}{|l|}{$\mathrm{AUC}_{\text {inf }}(\mathrm{h} \bullet \mathrm{ng} / \mathrm{mL})$} \\
\hline Mean (SD) & $2056.7(658.7)$ & $2069.8(656.1)$ & $1501.4(792.5)$ & $1106.2(753.0)$ \\
\hline Median (range) & $2150.9(864.8-2866.4)$ & $2189.7(801.3-3079.0)$ & $1451.4(371.6-3639.5)$ & $907.0(385.0-3414.0)$ \\
\hline \multicolumn{5}{|l|}{$C_{\max }(\mathrm{ng} / \mathrm{mL})$} \\
\hline Mean (SD) & $401.0(135.4)$ & $357.0(105.7)$ & $65.3(28.4)$ & $41.8(19.9)$ \\
\hline Median (range) & $411.3(115.7-582.7)$ & $358.9(121.9-523.3)$ & $64.9(20.5-113.2)$ & $37.9(18.1-92.2)$ \\
\hline \multicolumn{5}{|l|}{$t_{\max }(\mathrm{h})$} \\
\hline Mean (SD) & $1.9(0.9)$ & $1.8(0.7)$ & $7.8(2.4)$ & $10.0(2.9)$ \\
\hline Median (range) & $1.5(1.0-4.0)$ & $1.8(0.5-4.0)$ & $6.0(6.0-12.3)$ & $11.9(4.0-12.0)$ \\
\hline \multicolumn{5}{|l|}{$t_{1 / 2}(\mathrm{~h})$} \\
\hline Mean (SD) & $4.4(1.7)$ & $5.6(2.8)$ & $10.0(2.1)$ & $12.4(3.5)$ \\
\hline
\end{tabular}

$A U C_{i n f}$ area under the concentration-time curve from time zero to infinity, $B I D$ twice daily, $C_{\max }$ maximum observed concentration, $h$ hours, $S D$ standard deviation, $t_{1 / 2}$ apparent terminal elimination half-life, $t_{\max }$ time to maximum observed concentration

${ }^{a}$ Methotrexate (15-25 mg once weekly; $\left.15 \mathrm{mg}[n=10], 17.5 \mathrm{mg}[n=2], 20 \mathrm{mg}[n=2], 25 \mathrm{mg}[n=1]\right)$ was administered on Days 1 and 8 . Peficitinib (100 mg) was administered BID on Days 3-8, inclusive, and on the morning of Day 9

${ }^{\mathrm{b}}$ One patient experienced two serious adverse events and withdrew from the study; this patient did not receive peficitinib

4; methotrexate plus peficitinib, 6). One patient experienced an AE of sinus tachycardia in conjunction with SAEs of urinary tract infection and gastroenteritis; this patient discontinued before receiving peficitinib (see above). No other changes in vital signs were reported as AEs. No clinically significant electrocardiogram findings were observed. 
Table 4 Statistical analysis of pharmacokinetic interactions between peficitinib and methotrexate

Effect of peficitinib on the pharmacokinetics of methotrexate

\begin{tabular}{ll} 
Geometric mean $^{\mathrm{a}}$ & \\
\hline Methotrexate alone & Methotrexate + peficitinib \\
Day $1(n=14)^{\mathrm{b}}$ & Day $8(n=14)$
\end{tabular}

Geometric mean ratio

(90\% CI), \%

Day $1(n=14)^{\mathrm{b}} \quad$ Day $8(n=14)$

Methotrexate + pefi-

citinib/

methotrexate alone

\begin{tabular}{lccc}
\hline $\mathrm{AUC}_{\text {inf }}(\mathrm{h} \bullet \mathrm{ng} / \mathrm{mL})$ & 1899.9 & 1951.1 & $102.7(93.0-113.4)$ \\
$C_{\max }(\mathrm{ng} / \mathrm{mL})$ & 365.9 & 338.0 & $92.4(83.1-102.8)$ \\
Metabolite ratio $^{\mathrm{c}}$ & 0.67 & 0.48 & $72.7(65.6-80.5)$ \\
\hline
\end{tabular}

Effect of methotrexate on the pharmacokinetics of peficitinib

\begin{tabular}{lll} 
Geometric mean $^{\mathrm{a}}$ & & $\begin{array}{l}\text { Geometric mean ratio } \\
(90 \% \mathrm{CI}) \%\end{array}$ \\
\hline Peficitinib alone & Methotrexate + peficitinib & $\begin{array}{l}\text { Methotrexate + peficitinib/ } \\
\text { peficitinib alone }\end{array}$ \\
\hline Day 7 $(n=14)^{\mathrm{b}}$ & Day 8 $(n=14)$ & 980
\end{tabular}

$\begin{array}{llll}\mathrm{AUC}_{12}(\mathrm{~h} \bullet \mathrm{ng} / \mathrm{mL}) & 958.4 & 940.7 & 98.2(91.0-105.8)\end{array}$

$C_{\max }(\mathrm{ng} / \mathrm{mL}) \quad 255.5 \quad 234.9$

$92.0(78.2-108.1)$

$A U C_{12}$ area under the concentration-time curve within a 12-hour dosing interval, $A U C_{i n f}$ area under the concentration-time curve from time zero to infinity, $C I$ confidence interval, $C_{\max }$ maximum observed concentration, $h$ hours

${ }^{a}$ Exponentiated values of the least square mean from the mixed-effects model of the logarithm-transformed data

${ }^{b}$ Analysis was based on patients who completed the study; one patient experienced two serious adverse events and withdrew from the study; this patient did not receive peficitinib

${ }^{\mathrm{c}}$ The metabolite ratio was defined as $\mathrm{AUC}_{\mathrm{inf}}$ (7-hydroxymethotrexate)/AUC $\mathrm{Anf}_{\text {(methotrexate) }}$

Table 5 Summary of pharmacokinetic parameters for peficitinib

\begin{tabular}{|c|c|c|}
\hline Parameter & $\begin{array}{l}\text { Day } 7 \\
\text { Peficitinib alone }^{\mathrm{a}} \\
(n=14)\end{array}$ & $\begin{array}{l}\text { Day } 8 \\
\text { Peficitinib }+ \\
\text { methotrexate } \\
(n=14)^{\mathrm{b}}\end{array}$ \\
\hline \multicolumn{3}{|l|}{$\mathrm{AUC}_{12}(\mathrm{~h} \bullet \mathrm{ng} / \mathrm{mL})$} \\
\hline Mean (SD) & $1009.7(330.5)$ & $1009.4(366.2)$ \\
\hline Median (range) & $931.5(465.2-1645.6)$ & $984.3(386.6-1717.3)$ \\
\hline \multicolumn{3}{|l|}{$C_{\max }(\mathrm{ng} / \mathrm{mL})$} \\
\hline Mean (SD) & $266.3(78.8)$ & $253.2(87.9)$ \\
\hline Median (range) & $261.2(156.5-389.4)$ & $270.8(78.4-404.7)$ \\
\hline \multicolumn{3}{|l|}{$t_{\max }(\mathrm{h})$} \\
\hline Mean (SD) & $1.9(0.8)$ & $2.2(1.8)$ \\
\hline Median (range) & $1.5(1.0-3.0)$ & $1.8(0.6-8.1)$ \\
\hline \multicolumn{3}{|l|}{$C_{\text {trough, } \mathrm{AM}}(\mathrm{ng} / \mathrm{mL})$} \\
\hline Mean (SD) & $37.0(45.4)$ & $29.9(29.7)$ \\
\hline Median (range) & $18.5(2.2-178.5)$ & $17.9(3.3-97.5)$ \\
\hline
\end{tabular}

$A U C_{12}$ area under the concentration-time curve within a 12-hour dosing interval, $C_{\max }$ maximum observed concentration, $C_{\text {trough, } A M}$ trough plasma concentration in the morning, $h$ hours, $S D$ standard deviation, $t_{\text {max }}$ time to maximum observed concentration

${ }^{\text {a }}$ Methotrexate (15-25 mg once weekly; $15 \mathrm{mg}[n=10], 17.5 \mathrm{mg}$ $[n=2], 20 \mathrm{mg}[n=2], 25 \mathrm{mg}[n=1])$ was administered on Days 1 and 8. Peficitinib (100 mg) was administered BID on Days 3-8, inclusive, and on the morning of Day 9

${ }^{\mathrm{b}}$ One patient experienced two serious adverse events and withdrew from the study; this patient did not receive peficitinib

\section{Discussion}

This study indicates that peficitinib as a perpetrator had no significant effect on methotrexate pharmacokinetics when the two drugs were coadministered in patients with RA. Coadministration of methotrexate with peficitinib did decrease the metabolite ratio, defined as $\mathrm{AUC}_{\text {inf }}$ (7-hydroxymethotrexate/AUC ${ }_{\text {inf }}$ (methotrexate), by approximately $27 \%$; however, this observed decrease in 7-hydroxymethotrexate exposure in the presence of peficitinib appears unlikely to affect the efficacy of methotrexate. The inhibitory activity of methotrexate on dihydrofolic acid reductase, the mechanism whereby methotrexate inhibits cell proliferation [25], is 174fold more potent than that of 7-hydroxymethotrexate [26]. Methotrexate as a perpetrator had no significant effect on peficitinib AUC, and slightly decreased peficitinib $C_{\text {max }}$ by $8 \%$, which is not considered a clinically meaningful change.

The 2012 American College of Rheumatology guidelines on the use of DMARDs and biologic agents suggest that methotrexate monotherapy is not always sufficient to treat RA and combination therapy may be required [2]. Therefore, studies of drug-drug interactions of methotrexate with other RA treatments are important. Our in vitro studies of the effect of peficitinib on known membrane transporters of methotrexate showed that there were no significant inhibitory effects of peficitinib on MRP2, MRP4, or OAT1; however, peficitinib did inhibit BCRP and OAT3 
Table 6 Treatment-emergent adverse events

\begin{tabular}{|c|c|c|c|}
\hline Adverse events & $\begin{array}{l}\text { Methotrexate alone } \mathrm{e}^{\mathrm{a}} \\
(n=15)\end{array}$ & $\begin{array}{l}\text { Peficitinib alone } \mathrm{b}^{\mathrm{b}} \\
(n=14)\end{array}$ & $\begin{array}{l}\text { Methotrexate }+ \\
\text { peficitinib }^{c} \\
(n=14)\end{array}$ \\
\hline Patients who experienced $\geq 1 \mathrm{AE}^{\mathrm{d}}[n(\%)]$ & $5(33.3)$ & $7(50.0)$ & $7(50.0)$ \\
\hline $\begin{array}{l}\text { Patients who experienced AEs considered } \\
\text { to be related to the study } \operatorname{drug}^{\mathrm{d}}[n(\%)]\end{array}$ & $2(13.3)$ & $4(28.6)$ & $2(14.3)$ \\
\hline \multicolumn{4}{|l|}{ MedDRA preferred term $[n(\%)]$} \\
\hline Flatulence & 0 & $4(28.6)$ & $1(7.1)$ \\
\hline Muscle spasms & $1(6.7)$ & $2(14.3)$ & $1(7.1)$ \\
\hline Nausea & $2(13.3)$ & 0 & 0 \\
\hline Diarrhea & $1(6.7)$ & $1(7.1)$ & $1(7.1)$ \\
\hline Headache & $1(6.7)$ & 0 & $1(7.1)$ \\
\hline Oropharyngeal pain & 0 & $1(7.1)$ & $1(7.1)$ \\
\hline Eructation & 0 & $1(7.1)$ & $1(7.1)$ \\
\hline Borderline glaucoma & 0 & 0 & $1(7.1)$ \\
\hline Cataract & 0 & 0 & $1(7.1)$ \\
\hline Conjunctival hemorrhage & 0 & 0 & $1(7.1)$ \\
\hline Ocular hypertension & 0 & 0 & $1(7.1)$ \\
\hline Glossodynia & 0 & 0 & $1(7.1)$ \\
\hline Pharyngitis & 0 & 0 & $1(7.1)$ \\
\hline Urinary tract infection & 0 & 0 & $1(7.1)$ \\
\hline Retinal scar & 0 & 0 & $1(7.1)$ \\
\hline Dry mouth & 0 & $1(7.1)$ & 0 \\
\hline Abdominal distension & $1(6.7)$ & 0 & 0 \\
\hline Sinus tachycardia & $1(6.7)$ & 0 & 0 \\
\hline Dyspepsia & $1(6.7)$ & 0 & 0 \\
\hline Gastroesophageal reflux disease & $1(6.7)$ & 0 & 0 \\
\hline Vomiting & $1(6.7)$ & 0 & 0 \\
\hline Pyrexia & $1(6.7)$ & 0 & 0 \\
\hline Gastroenteritis $^{\mathrm{e}}$ & $1(6.7)$ & 0 & 0 \\
\hline
\end{tabular}

$A E$ adverse event, MedDRA Medical Dictionary for Regulatory Activities

${ }^{a}$ Methotrexate alone was Day 1 (after methotrexate dose) to Day 3 (before peficitinib dose)

${ }^{b}$ Peficitinib alone was Day 3 (after peficitinib dose) to Day 8 (before methotrexate dose)

${ }^{c}$ Methotrexate + peficitinib was Day 8 (after methotrexate dose) to the end of the study

${ }^{\mathrm{d}}$ Patients who experienced more than one AE were counted only once

${ }^{\mathrm{e}}$ The event of gastroenteritis was considered a serious AE that led to discontinuation from the study; one patient took only one dose of methotrexate and did not receive peficitinib. This patient also experienced a non-treatment-emergent serious AE of urinary tract infection before the first dose of methotrexate

with $\mathrm{IC}_{50}$ values of $13.5 \mu \mathrm{mol} / \mathrm{L}$ and $5.01 \mu \mathrm{mol} / \mathrm{L}$, respectively. In human plasma, $76-78 \%$ of peficitinib is bound to protein [27]. The current clinical study showed that the individual maximum plasma $C_{\max }$ of peficitinib after $100 \mathrm{mg}$ BID was $404.7 \mathrm{ng} / \mathrm{mL}$, which corresponds to a maximum protein-unbound $C_{\max }$ of approximately $93.1 \mathrm{ng} /$ $\mathrm{mL}(0.285 \mu \mathrm{mol} / \mathrm{L})$. The estimated maximum unbound $C_{\max }$ was consequently less than one-tenth of the $\mathrm{IC}_{50}$ for OAT3, which suggests a low likelihood of an inhibitory effect on renal excretion in vivo based on US Food and Drug Administration guidance [28].
Breast cancer resistance protein is present in the epithelium of the small intestine, colon, and kidney tubule [18, 21]. The estimated maximum $C_{\max }$ of unbound peficitinib in plasma $(0.285 \mu \mathrm{mol} / \mathrm{L})$ was approximately one-fiftieth of the $\mathrm{IC}_{50}$ for BCRP $(13.5 \mu \mathrm{mol} / \mathrm{L})$, suggesting a very low likelihood of an inhibitory effect on renal excretion in vivo based on US Food and Drug Administration guidance [28]. The theoretical maximum gastrointestinal peficitinib concentration (following a dose of $100 \mathrm{mg}$ of peficitinib dissolved in a volume of $250 \mathrm{~mL}$ of water) was estimated to be $0.4 \mathrm{mg} / \mathrm{mL}(1.23 \mathrm{mmol} / \mathrm{L})$, which was $>100$-fold of 
the in vitro $\mathrm{IC}_{50}$ for BCRP inhibition. This would suggest a possible intestinal drug-drug interaction between peficitinib and methotrexate based on US Food and Drug Administration guidance [28]. However, the pharmacokinetic measurements determined in the clinical study confirmed that coadministration of peficitinib and methotrexate had no significant effect on the pharmacokinetics of methotrexate in patients with RA. Furthermore, the clinical study showed that methotrexate had no significant effect on peficitinib AUC, although the $C_{\max }$ was decreased by $8 \%$. In a previous clinical study in patients with psoriasis, peficitinib $50 \mathrm{mg}$ once daily and $25 \mathrm{mg}$ BID had similar efficacy, as measured by the reduction from baseline in the Psoriasis Area and Severity Index and the Physician Static Global Assessment score [29]. Comparison of efficacy and pharmacokinetics from the psoriasis study suggests that the efficacy of peficitinib is unlikely to be associated with the $C_{\max }$. Therefore, the observed $8 \%$ decrease in $C_{\max }$ with methotrexate coadministration is not considered clinically relevant.

Methotrexate is largely excreted unchanged, with only partial oxidation by hepatic aldehyde oxidase to form 7-hydroxymethotrexate [17]. In contrast, peficitinib undergoes extensive metabolism via sulfate and methyl conjugation in the liver [14]. It is therefore unlikely that any metabolic drug-drug interactions contributed to the $27 \%$ reduction in 7-hydroxymethotrexate concentration and the $8 \%$ reduction in peficitinib $C_{\max }$ observed when peficitinib and methotrexate were coadministered.

The most frequently reported AEs associated with methotrexate alone include ulcerative stomatitis, leukopenia, nausea, and abdominal distress; other frequently reported AEs include malaise, fatigue, chills/fever, dizziness, and decreased resistance to infection [30]. Liver function test abnormalities also are frequently observed in patients taking methotrexate [31]. Findings from the current study suggest no additional or increased risk of these AEs with the addition of peficitinib to methotrexate.

In a pooled analysis of the safety of peficitinib from 13 phase I and II clinical trials in healthy volunteers and patients with psoriasis or RA, the AEs most often associated with peficitinib included headache, diarrhea, flatulence, neutropenia, nausea, abdominal pain, and vomiting [32]. In the present short-term study, no additional tolerability issues were observed with the combination of peficitinib and methotrexate. No abnormalities in laboratory findings, vital signs, or electrocardiogram results were reported. Moreover, two large phase III clinical trials have recently reported that peficitinib alone or in combination with methotrexate or other DMARDs was generally well tolerated over 52 weeks of treatment [23, 24], with a safety profile consistent with other currently available JAK inhibitors [33-35] and shortterm trials of peficitinib monotherapy $[32,36]$.
Previous studies have shown that tofacitinib did not significantly affect the pharmacokinetics of methotrexate and that methotrexate did not significantly affect the pharmacokinetics of tofacitinib [37], which is in line with the results of this study. The efficacy and tolerability of tofacitinib in combination with methotrexate in patients with RA have been previously reported [37-39].

Although a limitation of the current study design is that period effects will be confounded with treatment effects, the pharmacokinetics of neither peficitinib nor methotrexate showed time dependency. Additional limitations of the study include the small sample size $(N=15)$ and short duration, which may limit the ability of the study to accurately assess the true incidence of AEs. Finally, pharmacokinetic parameters for methotrexate were dose normalized because patients received individualized doses of methotrexate (ten patients, $15 \mathrm{mg}$; five patients, $>15$ to $\leq 25 \mathrm{mg}$ ), although it has been reported that the clearance of methotrexate is increased at higher plasma concentrations [40]. As the dose of methotrexate varied in this study, there is a possibility that methotrexate has a dose-dependent effect on lowering the $C_{\max }$ of peficitinib; however, visual examination of the methotrexate plasma concentration and dose-normalized plasma concentration shows few differences in concentration-time profiles.

\section{Conclusions}

Coadministration of peficitinib and methotrexate did not exhibit any new safety signals in this short-term clinical study, and coadministration had no clinically significant effect on the pharmacokinetic profile of either drug.

Acknowledgements Assistance with manuscript development was provided by Anny Wu, PharmD, and Kristine W. Schuler, MS, from Complete Healthcare Communications, Inc. (Chadds Ford, PA, USA), and Tina Patrick of Cello Health MedErgy (Europe). Kazuo Oda, PhD from Astellas Research Institute of America LLC and Miki Fujishima, MS of ADME \& Tox Research Institute, Sekisui Medical Co., Ltd performed the in vitro studies.

Author Contributions UV provided the study concept and study design, conducted the study, and was responsible for the data acquisition. UV and TZ analyzed and interpreted the data. TZ, SM, UV, YC, WZ, TS, $\mathrm{VC}$, and BA wrote and reviewed the manuscript.

\section{Compliance with Ethical Standards}

Funding Peficitinib is approved in Japan, Korea, and Taiwan for the treatment of rheumatoid arthritis. Astellas Pharma Global Development Inc. funded this study. Editorial assistance with manuscript development was funded by Astellas Pharma Inc.

Conflict of interest Tong Zhu, Udaya Valluri and Ying Cao are employees of Astellas Pharma Global Development Inc. Wenhui Zhang 
was an employee of Astellas Pharma Global Development Inc. at the time of the study. Selina Moy is an employee of Astellas Research Institute of America. Taiji Sawamoto is an employee of Astellas Pharma Inc. Bola Akinlade was an employee of Astellas Pharma Global Development Inc. at the time of the study, and is now an employee of Regeneron Pharmaceuticals; he reports no conflicts of interest that are directly relevant to the content of this study. Vishala Chindalore is an employee of Pinnacle Research Group/Anniston Medical Clinic, which received funding from Astellas to conduct the study.

Ethics Approval All procedures performed in studies involving human participants were in accordance with the ethical standards of the institutional review board for the study site (Quorum Review Institutional Review Board, Seattle, WA, USA) and with the 1964 Helsinki Declaration and its later amendments or comparable ethical standards.

Consent to Participate Informed consent was obtained from all individual participants included in the study.

Data Sharing Researchers may request access to anonymized participant -level data, trial-level data and protocols from Astellas-sponsored clinical trials at https://www.clinicalstudydatarequest.com. For the Astellas criteria on data sharing, see https://clinicalstudydatarequest.com/Study -Sponsors/Study-Sponsors-Astellas.aspx.

Open Access This article is licensed under a Creative Commons Attribution-NonCommercial 4.0 International License, which permits any non-commercial use, sharing, adaptation, distribution and reproduction in any medium or format, as long as you give appropriate credit to the original author(s) and the source, provide a link to the Creative Commons licence, and indicate if changes were made. The images or other third party material in this article are included in the article's Creative Commons licence, unless indicated otherwise in a credit line to the material. If material is not included in the article's Creative Commons licence and your intended use is not permitted by statutory regulation or exceeds the permitted use, you will need to obtain permission directly from the copyright holder. To view a copy of this licence, visit http://creativecommons.org/licenses/by-nc/4.0/.

\section{References}

1. Jacques P, Van den Bosch F. Emerging therapies for rheumatoid arthritis. Expert Opin Emerg Drugs. 2013;18:231-44.

2. Singh JA, Saag KG, Bridges SL, Akl EA, Bannuru RR, Sullivan MC, et al. 2015 American College of Rheumatology guideline for the treatment of rheumatoid arthritis. Arthritis Rheumatol. 2016;68:1-26.

3. Smolen JS, Landewé R, Bijlsma J, Burmester G, Chatzidionysiou $\mathrm{K}$, Dougados $\mathrm{M}$, et al. EULAR recommendations for the management of rheumatoid arthritis with synthetic and biological diseasemodifying antirheumatic drugs: 2016 update. Ann Rheum Dis. 2017;76:960-77.

4. Chakravarty SD, Poulikakos PI, Ivashkiv LB, Salmon JE, Kalliolias GD. Kinase inhibitors: a new tool for the treatment of rheumatoid arthritis. Clin Immunol. 2013;148:66-78.

5. O'Shea JJ, Kontzias A, Yamaoka K, Tanaka Y, Laurence A. Janus kinase inhibitors in autoimmune diseases. Ann Rheum Dis. 2013;72 Suppl. 2:ii111-15.

6. Jiang J, Ghoreschi K, Deflorian F, Chen Z, Perreira M, Pesu M, et al. Examining the chirality, conformation and selective kinase inhibition of 3-((3R,4R)-4-methyl-3-(methyl(7H-pyrrolo[2,3-d] pyrimidin-4-yl)amino)piperidin-1-yl)-3-oxopropanenitrile (CP690,550). J Med Chem. 2008;51:8012-8.

7. Stump KL, Lu LD, Dobrzanski P, Serdikoff C, Gingrich DE, Dugan BJ, et al. A highly selective, orally active inhibitor of Janus kinase 2, CEP-33779, ablates disease in two mouse models of rheumatoid arthritis. Arthritis Res Ther. 2011;13:R68.

8. Shuai K, Liu B. Regulation of JAK-STAT signalling in the immune system. Nat Rev Immunol. 2003;3:900-11.

9. Leonard WJ, Mitra S, Lin JX. JAK3 inhibition: is it sufficient? Nat Chem Biol. 2015;12:308-10.

10. Menet CJ, Van Rompaey L, Geney R. Advances in the discovery of selective JAK inhibitors. Prog Med Chem. 2013;52:153-223.

11. Higashi Y, Masamichi I, Ito M. ASP015K: a novel JAK inhibitor demonstrated potent efficacy in a chronic oxazolone-induced dermatitis model in rats. Dermatol Ther. 2012;2:S41.

12. Yamazaki S, Morio H, Inami M, Ito M, Fujii Y, Hanaoka K, et al. THU0101 ASP015K: a novel JAK inhibitor demonstrated potent efficacy in adjuvant-induced arthritis model in rats. Ann Rheum Dis. 2013;72:A197.

13. Taylor PC. Clinical efficacy of launched JAK inhibitors in rheumatoid arthritis. Rheumatology. 2019;58:i17-26.

14. Markham A, Keam SJ. Peficitinib: first global approval. Drugs. 2019;79:887-91.

15. Ito M, Yamazaki S, Yamagami K, Kuno M, Morita Y, Okuma $\mathrm{K}$, et al. A novel JAK inhibitor, peficitinib, demonstrates potent efficacy in a rat adjuvant-induced arthritis model. J Pharmacol Sci. 2017;133:25-33.

16. El-Sheikh AAK, van den Heuvel JJMW, Koenderink JB, Russel FGM. Interaction of nonsteroidal anti-inflammatory drugs with multidrug resistance protein (MRP) 2/ABCC2- and MRP4/ ABCC4-mediated methotrexate transport. J Pharmacol Exp Ther. 2007;320:229-35.

17. Bannwarth B, Péhourcq F, Schaeverbeke T, Dehais J. Clinical pharmacokinetics of low-dose pulse methotrexate in rheumatoid arthritis. Clin Pharmacokinet. 1996;30:194-210.

18. Mao Q, Unadkat JD. Role of the breast cancer resistance protein (BCRP/ABCG2) in drug transport: an update. AAPS J. 2015;17:65-82.

19. Takeda M, Khamdang S, Narikawa S, Kimura H, Hosoyamada $\mathrm{M}$, Cha SH, et al. Characterization of methotrexate transport and its drug interactions with human organic anion transporters. J Pharmacol Exp Ther. 2002;302:666-71.

20. Chioukh R, Noel-Hudson MS, Ribes S, Fournier N, Becquemont L, Verstuyft C. Proton pump inhibitors inhibit methotrexate transport by renal basolateral organic anion transporter hOAT3. Drug Metab Dispos. 2014;42:2041-8.

21. Maliepaard M, Scheffer GL, Faneyte IF, van Gastelen MA, Pijnenborg AC, Schinkel AH, et al. Subcellular localization and distribution of the breast cancer resistance protein transporter in normal human tissues. Cancer Res. 2001;61:3458-64.

22. Oda K, Cao YJ, Sawamoto T, Nakada N, Fisniku O, Nagasaka $\mathrm{Y}$, et al. Human mass balance, metabolite profile and identification of metabolic enzymes of [14C]ASP015K, a novel oral Janus kinase inhibitor. Xenobiotica. 2015;45:887-902.

23. Takeuchi T, Tanaka Y, Tanaka S, Kawakami A, Iwasaki M, Katayama K, et al. Efficacy and safety of peficitinib (ASP015K) in patients with rheumatoid arthritis and an inadequate response to methotrexate: results of a phase III randomised, double-blind, placebo-controlled trial (RAJ4) in Japan. Ann Rheum Dis. 2019;78:1305-19.

24. Tanaka Y, Takeuchi T, Tanaka S, Kawakami A, Iwasaki M, Song YW, et al. Efficacy and safety of peficitinib (ASP015K) in patients with rheumatoid arthritis and an inadequate response to conventional DMARDs: a randomised, double-blind, placebo-controlled phase III trial (RAJ3). Ann Rheum Dis. 2019;78:1320-32. 
25. Segal R, Yaron M, Tartakovsky B. Methotrexate: mechanism of action in rheumatoid arthritis. Semin Arthritis Rheum. 1990;20:190-200.

26. Farquhar D, Loo TL, Vadlamudi S. Synthesis and biologic evaluation of 7-hydroxymethotrexate, 7-methylaminopterin, and 7-methy lmethotrexate. J Med Chem. 1972;15:567-9.

27. Cao YJ, Sawamoto T, Valluri U, Cho K, Lewand M, Swan S, et al. Pharmacokinetics, pharmacodynamics, and safety of ASP015K (peficitinib), a new Janus kinase inhibitor, in healthy subjects. Clin Pharmacol Drug Dev. 2016;5:435-49.

28. US Food and Drug Administration. In vitro metabolism and transporter-mediated drug-drug interaction studies: guidance for industry. 2017. https://www.fda.gov/media/108130/download. Accessed 29 Aug 2019.

29. Papp K, Pariser D, Catlin M, Wierz G, Ball G, Akinlade B, et al. A phase 2a randomized, double-blind, placebo-controlled, sequential dose-escalation study to evaluate the efficacy and safety of ASP015K, a novel Janus kinase inhibitor, in patients with moderate-to-severe psoriasis. Br J Dermatol. 2015;173:767-76.

30. DAVA Pharmaceuticals Inc. Methotrexate tablets, USP. 2016. https://www.accessdata.fda.gov/drugsatfda_docs/label /2016/008085s066lbl.pdf. Accessed 19 Mar 2020.

31. Conway R, Carey JJ. Risk of liver disease in methotrexate treated patients. World J Hepatol. 2017;9:1092-100.

32. Garg J, Ball G, Cao Y, Zhu T, Tarzynski-Potempa R, Wisseh S. THU0227 pooled analysis of clinical safety data for ASP015K, a novel JAK inhibitor in development for treatment of autoimmune diseases. Ann Rheum Dis. 2013;72:241-2.

33. Dougados M, Van Der Heijde D, Chen Y-CC, Greenwald M, Drescher E, Liu J, et al. Baricitinib in patients with inadequate response or intolerance to conventional synthetic DMARDs: results from the RA-BUILD study. Ann Rheum Dis. 2017;76:88-95.
34. Atsumi T, Yamamoto K, Takeuchi T, Yamanaka H, Ishiguro N, Tanaka Y, et al. The first double-blind, randomised, parallel-group certolizumab pegol study in methotrexate-naïve early rheumatoid arthritis patients with poor prognostic factors, C-OPERA, shows inhibition of radiographic progression. Ann Rheum Dis. 2016;75:75-83.

35. Tanaka Y, Takeuchi T, Yamanaka H, Nakamura H, Toyoizumi $\mathrm{S}$, Zwillich S. Efficacy and safety of tofacitinib as monotherapy in Japanese patients with active rheumatoid arthritis: a 12-week, randomized, phase 2 study. Mod Rheumatol. 2015;25:514-21.

36. Takeuchi T, Tanaka Y, Iwasaki M, Ishikura H, Saeki S, Kaneko Y. Efficacy and safety of the oral Janus kinase inhibitor peficitinib (ASP015K) monotherapy in patients with moderate to severe rheumatoid arthritis in Japan: a 12-week, randomised, double-blind, placebo-controlled phase IIb study. Ann Rheum Dis. 2016;75:1057-64.

37. Cohen S, Zwillich SH, Chow V, Labadie RR, Wilkinson B. Coadministration of the JAK inhibitor CP-690,550 and methotrexate is well tolerated in patients with rheumatoid arthritis without need for dose adjustment. Br J Clin Pharmacol. 2010;69:143-51.

38. Kremer JM, Cohen S, Wilkinson BE, Connell CA, French JL, Gomez-Reino J, et al. A phase IIb dose-ranging study of the oral JAK inhibitor tofacitinib (CP-690,550) versus placebo in combination with background methotrexate in patients with active rheumatoid arthritis and an inadequate response to methotrexate alone. Arthritis Rheum. 2012;64:970-81.

39. Tanaka Y, Suzuki M, Nakamura H, Toyoizumi S, Zwillich SH. Phase II study of tofacitinib (CP-690,550) combined with methotrexate in patients with rheumatoid arthritis and an inadequate response to methotrexate. Arthritis Care Res. 2011;63:1150-8.

40. Hendel J, Nyfors A. Nonlinear renal elimination kinetics of methotrexate due to saturation of renal tubular reabsorption. Eur J Clin Pharmacol. 1984;26:121-4. 